\title{
Mappings of the Finite and Infinite Zero Structures and Invertibility Structures of General Linear Multivariable Systems Under the Bilinear Transformation
}

\author{
Ben M. Chen ${ }^{1}$ \\ Department of Electrical Engineering \\ National University of Singapore \\ Singapore 119260, Republic of Singapore \\ Email: bmchen@ee.nus.sg
}

\author{
Steven R. Weller \\ Department of Electrical \& Electronic Engineering \\ The University of Melbourne \\ Parkville, Victoria 3052, Australia \\ Email: s.weller@ee.mu.oz.au
}

Filename: papers/in-prepare/mu-bilinear-rev.tex, December 5, 2005

\begin{abstract}
This paper presents a comprehensive picture of the mapping of structural properties associated with general linear multivariable systems under bilinear transformation. While the mapping of poles of linear multivariable systems under such a transformation is well known, the question of how the structural invariant properties of a given system are mapped remains unanswered. This paper addresses this question. More specifically, we investigate how the finite and infinite zero structures, as well as invertibility structures, of a general continuous-time (discrete-time) linear time-invariant multivariable system are mapped to those of its discrete-time (continuous-time) counterpart under the bilinear (inverse bilinear) transformation. We demonstrate that the structural invariant indices lists $\mathcal{I}_{2}$ and $\mathcal{I}_{3}$ of Morse remain invariant under the bilinear transformation, while the structural invariant indices lists $\mathcal{I}_{1}$ and $\mathcal{I}_{4}$ of Morse are, in general, changed.
\end{abstract}

\section{Keywords:}

Linear system theory, continuous-time systems, discrete-time systems, bilinear transformation.

\footnotetext{
${ }^{1}$ The first author would like to acknowledge the University of Melbourne for offering him a visitorship from May 23 to June 16, 1996. This work was initiated and completed during his visit to the Department of Electrical \& Electronic Engineering, University of Melbourne.
} 


\section{Introduction and Problem Statement}

The need to perform continuous-time to discrete-time model conversions arises in a range of engineering contexts, including sampled-data control system design, and digital signal processing. As a consequence, numerous discretization procedures exist, including zero- and first-order hold input approximation, impulse invariant transformation, and bilinear transformation (see, for example Åström et al., 1984; and Franklin et al., 1980). Despite of the widespread use of the bilinear transform, however, a comprehensive treatment is lacking which details how key structural properties of continuous-time systems, such as the finite and infinite zero structures, and invertibility properties, are inherited by their discrete-time counterparts. Given the important role played by the infinite and finite zero structures in control system design, a clear understanding of the zero structures under bilinear transformation would be useful in the design of sampled-data control systems, and would complement existing results on the mapping of finite and infinite zero structures under zero-order hold sampling (see, for example, Åström et al., 1984; and Grizzle and Shor, 1988). In this paper, we present a comprehensive study of how the structures, i.e., the finite and infinite zero structures, as well as invertibility structures, of a general continuous-time (discrete-time) linear time-invariant system are mapped to those of its discrete-time (continuous-time) counterpart under the well known bilinear (inverse bilinear) transformations

$$
s=a\left(\frac{z-1}{z+1}\right) \quad \text { and } \quad z=\frac{a+s}{a-s},
$$

respectively. Since the bilinear and inverse bilinear transformations find widespread use in digital control and signal processing, the results obtained have immediate applications. In particular, some of the results have already been applied to solve discrete-time algebraic Riccati equations (see for example Chen et al., 1994), and in the solution of certain $H_{\infty}$ control problems (see for example, Chen, 1996; and Chen et al., 1996).

We consider in this paper general linear time-invariant systems characterized by

$$
\Sigma:\left\{\begin{aligned}
\delta x & =A x+B u, \\
y & =C x+D u,
\end{aligned}\right.
$$

where $x \in \mathbb{R}^{n}, y \in \mathbb{R}^{p}, u \in \mathbb{R}^{m}$ and $A, B, C$ and $D$ are matrices of appropriate dimensions. In (1.2), $\delta$ is an operator defined as follows: $\delta x=\dot{x}$ if $\Sigma$ is a continuous-time system, and $\delta x=x(k+1)$ if $\Sigma$ is a discrete-time system. Without loss of any generality, we assume throughout this paper that both matrices $\left[\begin{array}{ll}C & D\end{array}\right]$ and $\left[\begin{array}{ll}B^{\prime} & D^{\prime}\end{array}\right]$ are of full rank.

The paper is organized as follows: In Section 2, we recall the special coordinate basis of linear systems, which is instrumental to the development and derivation of the main results. Section 3 contains the main results of this paper, namely, in Subsection 3.1 we present the mapping of finite and infinite zero structures of a continuous-time linear time invariant system to those of its discrete-time counterpart under the bilinear transformation, while in Subsection 3.2, we give corresponding results for the mapping from discrete-time to continuous-time. Section 4 gives the detailed proof of our main results. Finally, concluding remarks are made in Section 5.

Throughout this paper, $X^{\prime}$ denotes the transpose of matrix $X$. I denotes an identity matrix of appropriate dimension, while $I_{k}$ denotes a $k \times k$ identity matrix. $\mathbb{R}$ and $\mathbb{C}$ are respectively the sets of real and complex numbers. $\lambda(X)$ is the set of eigenvalues of a real square matrix $X$. 


\section{Background Materials}

In this section, we recall the special coordinate basis of linear systems from Sannuti and Saberi (1987), and Saberi and Sannuti (1990), which is instrumental to the main results developed in subsequent sections. We will also recall some basic concepts of finite and infinite zero structures of linear time invariant systems. First, it can be easily shown using the singular value decomposition that one can always find nonsingular matrices $U$ and $V$ that put the direct feedthrough matrix $D$ of (1.2) into the following form

$$
U D V=\left[\begin{array}{cc}
I_{m_{0}} & 0 \\
0 & 0
\end{array}\right]
$$

where $m_{0}$ is the rank of $D$. It is well known that these nonsingular transformations $V$ and $U$ leave the finite and infinite zero structures of $\Sigma$ unaffected. Hence, without loss of generality one can assume that the matrix $D$ in (1.2) has the form as shown in (2.1). Thus the system in (1.2) can be rewritten as

$$
\left\{\begin{array}{c}
\delta x=A \quad x+\left[\begin{array}{ll}
B_{0} & B_{1}
\end{array}\right]\left(\begin{array}{l}
u_{0} \\
u_{1}
\end{array}\right), \\
\left(\begin{array}{l}
y_{0} \\
y_{1}
\end{array}\right)=\left[\begin{array}{l}
C_{0} \\
C_{1}
\end{array}\right] x+\left[\begin{array}{cc}
I_{m_{0}} & 0 \\
0 & 0
\end{array}\right]\left(\begin{array}{l}
u_{0} \\
u_{1}
\end{array}\right),
\end{array}\right.
$$

where $B_{0}, B_{1}, C_{0}$ and $C_{1}$ are the matrices of appropriate dimensions. We have the following theorem.

Theorem 2.1. For any given system $\Sigma$ of (1.2), there exist

1. coordinate-free non-negative integers $n_{a}, n_{b}, n_{c}, n_{d}, m_{d} \leq m-m_{0}$ and $q_{i}, i=1,2, \cdots, m_{d}$;

2. nonsingular state, output, and input transformations $\Gamma_{s}, \Gamma_{o}$ and $\Gamma_{i}$ which take the given $\Sigma$ into a special coordinate basis that explicitly displays both finite and infinite zero structures of $\Sigma$.

The special coordinate basis is described by the following set of equations:

$$
\begin{gathered}
u=\Gamma_{i}\left(\begin{array}{c}
u_{0} \\
u_{d} \\
u_{c}
\end{array}\right), \quad x=\Gamma_{s}\left(\begin{array}{c}
x_{a} \\
x_{b} \\
x_{c} \\
x_{d}
\end{array}\right), \quad y=\Gamma_{o}\left(\begin{array}{c}
y_{0} \\
y_{d} \\
y_{b}
\end{array}\right), \\
x_{d}=\left(\begin{array}{c}
x_{1} \\
\vdots \\
x_{m_{d}}
\end{array}\right), \quad u_{d}=\left(\begin{array}{c}
u_{1} \\
\vdots \\
u_{m_{d}}
\end{array}\right), \quad y_{d}=\left(\begin{array}{c}
y_{1} \\
\vdots \\
y_{m_{d}}
\end{array}\right),
\end{gathered}
$$

and

$$
\begin{gathered}
\delta x_{a}=A_{a a} x_{a}+B_{a 0} y_{0}+L_{a d} y_{d}+L_{a b} y_{b}, \\
\delta x_{b}=A_{b b} x_{b}+B_{b 0} y_{0}+L_{b d} y_{d}, \quad y_{b}=C_{b} x_{b}, \\
\delta x_{c}=A_{c c} x_{c}+B_{c 0} y_{0}+L_{c d} y_{d}+L_{c b} y_{b}+B_{c} E_{c a} x_{a}+B_{c} u_{c} \\
y_{0}=C_{0 a} x_{a}+C_{0 b} x_{b}+C_{0 c} x_{c}+C_{0 d} x_{d}+u_{0},
\end{gathered}
$$


and for $i=1,2, \cdots, m_{d}$,

$$
\begin{gathered}
\delta x_{i}=A_{q_{i}} x_{i}+L_{i 0} y_{0}+L_{i d} y_{d}+B_{q_{i}}\left[u_{i}+E_{i a} x_{a}+E_{i b} x_{b}+E_{i c} x_{c}+E_{i d} x_{d}\right] \\
y_{i}=C_{q_{i}} x_{i}, \quad y_{d}=C_{d} x_{d} .
\end{gathered}
$$

Here the states $x_{a}, x_{b}, x_{c}$ and $x_{d}$ are respectively of dimensions $n_{a}, n_{b}, n_{c}$ and $n_{d}=\sum_{i=1}^{m_{d}} q_{i}$, while $x_{i}$ is of dimension $q_{i}$ for each $i=1,2, \cdots, m_{d}$. The control vectors $u_{0}, u_{d}$ and $u_{c}$ are respectively of dimensions $m_{0}, m_{d}$ and $m_{c}=m-m_{0}-m_{d}$ while the output vectors $y_{0}, y_{d}$ and $y_{b}$ are respectively of dimensions $m_{0}, m_{d}$ and $p_{b}=p-m_{0}-m_{d}$. The matrices $A_{q_{i}}, B_{q_{i}}$ and $C_{q_{i}}$ have the following form:

$$
A_{q_{i}}=\left[\begin{array}{cc}
0 & I_{q_{i}-1} \\
0 & 0
\end{array}\right], \quad B_{q_{i}}=\left[\begin{array}{l}
0 \\
1
\end{array}\right], \quad C_{q_{i}}=\left[\begin{array}{llll}
1 & 0 & \cdots & 0
\end{array}\right] .
$$

Furthermore, the pair $\left(A_{c c}, B_{c}\right)$ is controllable and the pair $\left(A_{b b}, C_{b}\right)$ is observable.

Proof: The original work of the special coordinate basis was reported in Sannuti and Saberi (1987) and Saberi and Sannuti (1990). Software packages that realize the above special coordinate basis are available either in LAS by Chen (1988) or in MATLAB by Lin (1989).

For future use, we rewrite the special coordinate basis given in Theorem 2.1 in a more compact form as a system characterized by the quadruple $\left(A_{\mathrm{SCB}}, B_{\mathrm{SCB}}, C_{\mathrm{SCB}}, D_{\mathrm{SCB}}\right)$, where

$$
\begin{gathered}
A_{\mathrm{SCB}}=: \Gamma_{s}^{-1}\left(A-B_{0} C_{0}\right) \Gamma_{s}=\left[\begin{array}{cccc}
A_{a a} & L_{a b} C_{b} & 0 & L_{a d} C_{d} \\
0 & A_{b b} & 0 & L_{b d} C_{d} \\
B_{c} E_{c a} & L_{c b} C_{b} & A_{c c} & L_{c d} C_{d} \\
B_{d} E_{d a} & B_{d} E_{d b} & B_{d} E_{d c} & A_{d d}
\end{array}\right] \\
B_{\mathrm{SCB}}:=\Gamma_{s}^{-1}\left[\begin{array}{ll}
B_{0} & B_{1}
\end{array}\right] \Gamma_{i}=\left[\begin{array}{ccc}
B_{a 0} & 0 & 0 \\
B_{b 0} & 0 & 0 \\
B_{c 0} & 0 & B_{c} \\
B_{d 0} & B_{d} & 0
\end{array}\right]
\end{gathered}
$$

and

$$
C_{\mathrm{SCB}}:=\Gamma_{o}^{-1}\left[\begin{array}{l}
C_{0} \\
C_{1}
\end{array}\right] \Gamma_{s}=\left[\begin{array}{cccc}
C_{0 a} & C_{0 b} & C_{0 c} & C_{0 d} \\
0 & 0 & 0 & C_{d} \\
0 & C_{b} & 0 & 0
\end{array}\right], \quad D_{\mathrm{SCB}}:=\Gamma_{o}^{-1} D \Gamma_{i}=\left[\begin{array}{ccc}
I_{m_{0}} & 0 & 0 \\
0 & 0 & 0 \\
0 & 0 & 0
\end{array}\right] .
$$

In what follows, we recall some properties of the special coordinate basis that are pertinent to the present work. The first property we will recall deals with the invariant zeros and their structures of the given systems (see also MacFarlane and Karcanias, 1976; and Saberi et al., 1991; for details).

Property 2.1. Invariant zeros of $\Sigma$ are the eigenvalues of $A_{a a}$, i.e., $\lambda\left(A_{a a}\right)$.

In order to display various multiplicities of invariant zeros, let $X_{a}$ be a nonsingular transformation matrix such that

$$
X_{a}^{-1} A_{a a} X_{a}=J=\text { Block diag }\left\{J_{1}, J_{2}, \cdots, J_{k}\right\},
$$


where $J_{i}, i=1,2, \cdots, k$, are some $n_{i} \times n_{i}$ Jordan blocks:

$$
J_{i}=\operatorname{diag}\left\{\alpha_{i}, \alpha_{i}, \cdots, \alpha_{i}\right\}+\left[\begin{array}{cc}
0 & I_{n_{i}-1} \\
0 & 0
\end{array}\right] .
$$

For any given $\alpha \in \lambda\left(A_{a a}\right)$, let there be $\tau_{\alpha}$ Jordan blocks of $A_{a a}$ associated with $\alpha$. Let $n_{\alpha, 1}, n_{\alpha, 2}$, $\cdots, n_{\alpha, \tau_{\alpha}}$ be the dimensions of the corresponding Jordan blocks. Then we say $\alpha$ is an invariant zero of $\Sigma$ with multiplicity structure $S_{\alpha}^{\star}(\Sigma)$ (see also Saberi et al., 1991),

$$
S_{\alpha}^{\star}(\Sigma)=\left\{n_{\alpha, 1}, n_{\alpha, 2}, \cdots, n_{\alpha, \tau_{\alpha}}\right\} .
$$

The geometric multiplicity of $\alpha$ is then simply given by $\tau_{\alpha}$, and the algebraic multiplicity of $\alpha$ is given by $\sum_{i=1}^{\tau_{\alpha}} n_{\alpha, i}$. Here we should note that the invariant zeros together with their structures of $\Sigma$ are related to the structural invariant indices list $\mathcal{I}_{1}(\Sigma)$ of Morse (1973).

The next property concerns the infinite structure of the given system, which is related to the structural invariant indices list $\mathcal{I}_{4}(\Sigma)$ of Morse (see, for example, Commault and Dion, 1982; Morse, 1973; Owens, 1978; and Saberi et al., 1995; for relevant definitions).

Property 2.2. $\Sigma$ has $m_{0}=\operatorname{rank}(D)$ infinite zeros of order 0 . The infinite zero structure (of order greater than 0 ) of $\Sigma$ is given by

$$
S_{\infty}^{\star}(\Sigma)=\left\{q_{1}, q_{2}, \cdots, q_{m_{d}}\right\} .
$$

That is, each $q_{i}$ corresponds to an infinite zero of $\Sigma$ of order $q_{i}$.

Here we would note that the index $\mathcal{S}_{\infty}^{\star}$ of $(2.18)$ is not exactly the same as $\mathcal{I}_{4}$ of Morse. However, they are related and can be converted to one another in a trivial manner (see Saberi et al., 1995).

The special coordinate basis can also exhibit the invertibility structure of a given system $\Sigma$. The definitions of right and left invertibility of a linear system can be found in Moylan (1977).

Property 2.3. The given system $\Sigma$ is right invertible if and only if $x_{b}$ (and hence $y_{b}$ ) are nonexistent, left invertible if and only if $x_{c}$ (and hence $u_{c}$ ) are non-existent, and invertible if and only if both $x_{b}$ and $x_{c}$ are non-existent. Moreover, $\Sigma$ is degenerate if and only if it is neither left nor right invertible, i.e., both $x_{b}$ and $x_{c}$ are present.

The special coordinate basis can also be modified to obtain the structural invariant indices lists $\mathcal{I}_{2}$ and $\mathcal{I}_{3}$ of Morse (1973) of the given system $\Sigma$. In order to display $\mathcal{I}_{2}(\Sigma)$, we let $X_{c}$ and $X_{i}$ be nonsingular matrices such that the controllable pair $\left(A_{c c}, B_{c}\right)$ is transformed into Brunovsky canonical form, i.e.,

$$
X_{c}^{-1} A_{c c} X_{c}=\left[\begin{array}{ccccc}
0 & I_{\ell_{1}-1} & \cdots & 0 & 0 \\
\star & \star & \cdots & \star & \star \\
\vdots & \vdots & \ddots & \vdots & \vdots \\
0 & 0 & \cdots & 0 & I_{\ell_{m_{c}}-1} \\
\star & \star & \cdots & \star & \star
\end{array}\right], \quad X_{c}^{-1} B_{c} X_{i}=\left[\begin{array}{ccc}
0 & \cdots & 0 \\
1 & \cdots & 0 \\
\vdots & \ddots & \vdots \\
0 & \cdots & 0 \\
0 & \cdots & 1
\end{array}\right]
$$


where $\star$ 's denote constant scalars or row vectors. Then we have

$$
\mathcal{I}_{2}(\Sigma)=\left\{\ell_{1}, \cdots, \ell_{m_{c}}\right\}
$$

which is also called the controllability index of $\left(A_{c c}, B_{c}\right)$. Similarly, we have

$$
\mathcal{I}_{3}(\Sigma)=\left\{\mu_{1}, \cdots, \mu_{p_{b}}\right\}
$$

where $\left\{\mu_{1}, \cdots, \mu_{p_{b}}\right\}$ is the controllability index of the controllable pair $\left(A_{b b}^{\prime}, C_{b}^{\prime}\right)$.

\section{A Complete Structural Picture of the Bilinear Transformation}

We present our main results in this section. More specifically, in Subsection 3.1, we study how the finite and infinite zero structures, as well as invertibility properties, of a continuous-time linear time-invariant system are mapped to those of its discrete-time counterpart under bilinear transformation. In Subsection 3.2, we present corresponding results for the mapping from a discrete-time to continuous-time. Two examples are included to illustrate the results obtained in this section. The detailed proof of our main results, i.e., Theorem 3.1, will be given in the next section for the sake of clarity.

\subsection{From Continuous-time to Discrete-time}

The bilinear transformation has been widely applied to solve problems in the areas of signal processing, communications and control system design. It is therefore important to study its structural behavior in order to have a clear understanding of this technique. In this subsection, we will consider a continuous-time linear time-invariant system $\Sigma_{c}$ characterized by

$$
\Sigma_{c}:\left\{\begin{array}{l}
\dot{x}=A x+B u \\
y=C x+D u
\end{array}\right.
$$

where $\Sigma_{c}$ has transfer function

$$
G_{c}(s)=C(s I-A)^{-1} B+D .
$$

Let us apply a bilinear transformation to the above continuous-time system, by replacing $s$ in (3.2) with

$$
s=\frac{2}{T}\left(\frac{z-1}{z+1}\right)=a\left(\frac{z-1}{z+1}\right)
$$

where $T=2 / a$ is the sampling period. As presented in (3.3), the bilinear transformation is often called Tustin's approximation, while the choice

$$
a=\frac{\omega_{1}}{\tan \left(\omega_{1} T / 2\right)}
$$

yields the pre-warped Tustin approximation, in which the frequency responses of the continuoustime system and its discrete-time counterpart are matched at frequency $\omega_{1}$. In this way, we obtain a discrete-time system

$$
G_{d}(z)=C\left(a \frac{z-1}{z+1} I-A\right)^{-1} B+D .
$$


The following lemma provides a direct state-space realization of $G_{d}(z)$. Its proof is straightforward and hence is omitted.

Lemma 3.1. A state-space realization of $G_{d}(z)$, the discrete-time counterpart of the continuoustime system $\Sigma_{c}$ of (3.1) under the bilinear transformation (3.3), is given by

$$
\Sigma_{d}:\left\{\begin{aligned}
x(k+1) & =\tilde{A} x(k)+\tilde{B} u(k), \\
y(k) & =\tilde{C} x(k)+\tilde{D} u(k),
\end{aligned}\right.
$$

where

$$
\left.\begin{array}{l}
\tilde{A}=(a I+A)(a I-A)^{-1}, \\
\tilde{B}=\sqrt{2 a}(a I-A)^{-1} B, \\
\tilde{C}=\sqrt{2 a} C(a I-A)^{-1}, \\
\tilde{D}=D+C(a I-A)^{-1} B .
\end{array}\right\}
$$

Here we clearly assume that matrix $A$ has no eigenvalues at $a$.

The following theorem establishes the interconnection of the structural properties of $\Sigma_{c}$ and $\Sigma_{d}$, and forms the major contribution of this paper.

Theorem 3.1. Consider the continuous-time system $\Sigma_{c}$ of (3.1) characterized by the quadruple $(A, B, C, D)$ with matrix $A$ having no eigenvalues at $a$, and its discrete-time counterpart under the bilinear transformation (3.3), i.e., $\Sigma_{d}$ of (3.6) characterized by the quadruple $(\tilde{A}, \tilde{B}, \tilde{C}, \tilde{D})$. We have the following properties:

1. Controllability (stabilizability) and observability (detectability) of $\Sigma_{d}$

(a) $\Sigma_{d}$ is controllable (stabilizable) if and only if $\Sigma_{c}$ is controllable (stabilizable).

(b) $\Sigma_{d}$ is observable (detectable) if and only if $\Sigma_{c}$ is observable (detectable).

2. Effects of nonsingular state, output and input transformations, together with state feedback and output injection laws

(a) For any given nonsingular state, output and input transformations $T_{s}, T_{o}$ and $T_{i}$, the quadruple $\left(T_{s}^{-1} \tilde{A} T_{s}, T_{s}^{-1} \tilde{B} T_{i}, T_{o}^{-1} \tilde{C} T_{s}, T_{o}^{-1} \tilde{D} T_{i}\right)$, is the discrete-time counterpart of the continuous time system $\left(T_{s}^{-1} A T_{s}, T_{s}^{-1} B T_{i}, T_{o}^{-1} C T_{s}, T_{o}^{-1} D T_{i}\right)$ under the transformation (3.3).

(b) For any $F \in \mathbb{R}^{m \times n}$ with $A+B F$ having no eigenvalues at $a$, define a nonsingular matrix

$$
\tilde{T}_{i}:=I+F(a I-A-B F)^{-1} B=\left[I-F(a I-A)^{-1} B\right]^{-1} \in \mathbb{R}^{m \times m}
$$

and a constant matrix

$$
\tilde{F}:=\sqrt{2 a} F(a I-A-B F)^{-1} \in \mathbb{R}^{m \times n} .
$$

Then a continuous-time system $\Sigma_{c F}$ characterized by $(A+B F, B, C+D F, D)$ is mapped to a discrete-time system $\Sigma_{d \mathrm{~F}}$, characterized by $\left(\tilde{A}+\tilde{B} \tilde{F}, \tilde{B} \tilde{T}_{i}, \tilde{C}+\tilde{D} \tilde{F}, \tilde{D} \tilde{T}_{i}\right)$, under the bilinear transformation (3.3). 
(c) For any $K \in \mathbb{R}^{n \times p}$ with $A+K C$ having no eigenvalues at $a$, define a nonsingular matrix

$$
\tilde{T}_{o}:=\left[I+C(a I-A-K C)^{-1} K\right]^{-1} \in \mathbb{R}^{p \times p}
$$

and a constant matrix

$$
\tilde{K}:=\sqrt{2 a}(a I-A-K C)^{-1} K .
$$

Then a continuous-time system $\Sigma_{c K}$ characterized by $(A+K C, B+K D, C, D)$ is mapped to a discrete-time system $\Sigma_{d \mathrm{~K}}$, characterized by $\left(\tilde{A}+\tilde{K} \tilde{C}, \tilde{B}+\tilde{K} \tilde{D}, \tilde{T}_{o}^{-1} \tilde{C}, \tilde{T}_{o}^{-1} \tilde{D}\right)$, under the bilinear transformation (3.3).

3. Invertibility and structural invariant indices lists $\mathcal{I}_{2}$ and $\mathcal{I}_{3}$ of $\Sigma_{d}$

(a) $\mathcal{I}_{2}\left(\Sigma_{d}\right)=\mathcal{I}_{2}\left(\Sigma_{c}\right)$, and $\mathcal{I}_{3}\left(\Sigma_{d}\right)=\mathcal{I}_{3}\left(\Sigma_{c}\right)$.

(b) $\Sigma_{d}$ is left (right) invertible if and only if $\Sigma_{c}$ is left (right) invertible.

(c) $\Sigma_{d}$ is invertible (degenerate) if and only if $\Sigma_{c}$ is invertible (degenerate).

4. The invariant zeros of $\Sigma_{d}$ and their associated structures consist of the following two parts:

(a) Let the infinite zero structure (of order greater than 0) of $\Sigma_{c}$ be given by $S_{\infty}^{\star}\left(\Sigma_{c}\right)=$ $\left\{q_{1}, q_{2}, \cdots, q_{m_{d}}\right\}$. Then $z=-1$ is an invariant zero of $\Sigma_{d}$ with multiplicity structure $S_{-1}^{\star}\left(\Sigma_{d}\right)=\left\{q_{1}, q_{2}, \cdots, q_{m_{d}}\right\}$.

(b) Let $s=\alpha \neq a$ be an invariant zero of $\Sigma_{c}$ with multiplicity structure $S_{\alpha}^{\star}\left(\Sigma_{c}\right)=$ $\left\{n_{\alpha, 1}, n_{\alpha, 2}, \cdots, n_{\alpha, \tau_{\alpha}}\right\}$. Then $z=\beta=(a+\alpha) /(a-\alpha)$ is an invariant zero of $\Sigma_{d}$ with multiplicity structure $S_{\beta}^{\star}\left(\Sigma_{d}\right)=\left\{n_{\alpha, 1}, n_{\alpha, 2}, \cdots, n_{\alpha, \tau_{\alpha}}\right\}$.

5. The infinite zero structure of $\Sigma_{d}$ consists of the following two parts:

(a) Let $m_{0}$ be the number of infinite zeros of $\Sigma_{c}$ of order 0 , i.e., $m_{0}=\operatorname{rank}(D)$, and let $m_{d}$ be the total number of infinite zeros of $\Sigma_{c}$ of order greater than 0 . Also, let $\tau_{a}$ be the geometric multiplicity of the invariant zero of $\Sigma_{c}$ at $s=a$. Then the total number of infinite zeros of $\Sigma_{d}$ of order 0, i.e., rank $(\tilde{D})$, is equal to $m_{0}+m_{d}-\tau_{a}$.

(b) Let $s=a$ be an invariant zero of the given continuous-time system $\Sigma_{c}$ with a multiplicity structure $S_{a}^{\star}\left(\Sigma_{c}\right)=\left\{n_{a, 1}, n_{a, 2}, \cdots, n_{a, \tau_{a}}\right\}$. Then $\Sigma_{d}$ has an infinite zero (of order greater than 0) structure $S_{\infty}^{\star}\left(\Sigma_{d}\right)=\left\{n_{a, 1}, n_{a, 2}, \cdots, n_{a, \tau_{a}}\right\}$.

We have the following two interesting observations. The first is with regard to the minimum phase and nonminimum phase properties of $\Sigma_{d}$, while the second concerns the asymptotic behavior of $\Sigma_{d}$ as the sampling period $T$ tends to zero (or, equivalently, as $a \rightarrow \infty$ ).

Observation 3.1. Consider a general continuous-time system $\Sigma_{c}$ and its discrete-time counterpart $\Sigma_{d}$ under the transformation (3.3). It follows from 4(a) and 4(b) of Theorem 3.1 that

1. $\Sigma_{d}$ has all its invariant zeros inside the unit circle if and only if $\Sigma_{c}$ has all its invariant zeros in the open left-half plane; 
2. $\Sigma_{d}$ has invariant zeros on the unit circle if and only if $\Sigma_{c}$ has invariant zeros on the imaginary axis, and/or $\Sigma_{c}$ has at least one infinite zero of order greater than 0 ;

3. $\Sigma_{d}$ has invariant zeros outside the unit circle if and only if $\Sigma_{c}$ has invariant zeros in the open right-half plane.

Observation 3.2. Consider a general continuous-time system $\Sigma_{c}$ and its discrete-time counterpart $\Sigma_{d}$ under the bilinear transformation (3.3). Then a consequence of Theorem 3.1, $\Sigma_{d}$ has the following asymptotic properties as the sampling period $T$ tends to zero (but not equal to zero):

1. $\Sigma_{d}$ has no infinite zeros of order greater than 0 , i.e., no delays from the input to the output;

2. $\Sigma_{d}$ has one invariant zero at $z=-1$ with an appropriate multiplicity structure if $\Sigma_{c}$ has any infinite zeros of order greater than 0 ; and

3. The remaining invariant zeros of $\Sigma_{d}$, if any, tend to the point $z=1$. More interestingly, the invariant zeros of $\Sigma_{d}$ corresponding to the stable invariant zeros of $\Sigma_{c}$ are always stable, and approach the point $z=1$ from inside the unit circle. Conversely, the invariant zeros of $\Sigma_{d}$ corresponding to the unstable invariant zeros of $\Sigma_{c}$ are always unstable, and approach the point $z=1$ from outside the unit circle. Finally, those associated with the imaginary axis invariant zeros of $\Sigma_{c}$ are always mapped onto the unit circle and move towards to the point $z=1$.

\subsection{From Discrete-time to Continuous-time}

The inverse bilinear transformation has found an important application in the solution of discretetime $H_{\infty}$ control problems. Namely, one can design a discrete-time $H_{\infty}$ suboptimal controller by (i) first transforming the discrete-time model to continuous-time, using an inverse bilinear transformation; (ii) designing a continuous-time $H_{\infty}$ suboptimal controller for the continuoustime model so obtained; and then (iii) the discrete-time $H_{\infty}$ suboptimal controller is given by the bilinear transformation of the obtained continuous-time $H_{\infty}$ suboptimal controller. The inverse bilinear transformation has also been applied to solve general discrete-time Riccati equations (see Chen et al., 1994).

We present in this subsection a similar result as in the previous subsection, but for the inverse bilinear transformation mapping a discrete-time system to a continuous-time system. We begin with a discrete-time linear time-invariant system $\tilde{\Sigma}_{d}$ characterized by

$$
\tilde{\Sigma}_{d}:\left\{\begin{aligned}
x(k+1) & =\tilde{A} x(k)+\tilde{B} u(k), \\
y(k) & =\tilde{C} x(k)+\tilde{D} u(k),
\end{aligned}\right.
$$

and with corresponding transfer function

$$
H_{d}(z)=\tilde{C}(z I-\tilde{A})^{-1} \tilde{B}+\tilde{D} .
$$

The inverse bilinear transformation corresponding to (3.3) replaces $z$ in (3.13) with

$$
z=\frac{a+s}{a-s}
$$


to obtain the following continuous-time system:

$$
H_{c}(s)=\tilde{C}\left(\frac{a+s}{a-s} I-\tilde{A}\right)^{-1} \tilde{B}+\tilde{D}
$$

The following lemma is analogous to Lemma 3.1, and provides a state-space realization of $H_{c}(s)$.

Lemma 3.2. A state-space realization of $H_{c}(s)$, the continuous-time counterpart of the discretetime system $\tilde{\Sigma}_{d}$ of (3.12) under the inverse bilinear transformation (3.14), is given by

$$
\tilde{\Sigma}_{c}:\left\{\begin{array}{l}
\dot{x}=A x+B u \\
y=C x+D u
\end{array}\right.
$$

where

$$
\begin{aligned}
& A=a(\tilde{A}+I)^{-1}(\tilde{A}-I), \\
& B=\sqrt{2 a}(\tilde{A}+I)^{-1} \tilde{B}, \\
& C=\sqrt{2 a} \tilde{C}(\tilde{A}+I)^{-1}, \\
& D=\tilde{D}-\tilde{C}(\tilde{A}+I)^{-1} \tilde{B} .
\end{aligned}
$$

Here we clearly assume that the matrix $\tilde{A}$ has no eigenvalues at -1 .

The following theorem is analogous to Theorem 3.1.

Theorem 3.2. Consider the discrete-time system $\tilde{\Sigma}_{d}$ of (3.12) characterized by the quadruple $(\tilde{A}, \tilde{B}, \tilde{C}, \tilde{D})$ with matrix $\tilde{A}$ having no eigenvalues at -1 , and its continuous-time counterpart under the inverse bilinear transformation (3.14), i.e., $\tilde{\Sigma}_{c}$ of (3.16) characterized by the quadruple $(A, B, C, D)$. We have the following properties:

1. Controllability (stabilizability) and observability (detectability) of $\tilde{\Sigma}_{c}$

(a) $\tilde{\Sigma}_{c}$ is controllable (stabilizable) if and only if $\tilde{\Sigma}_{d}$ is controllable (stabilizable).

(b) $\tilde{\Sigma}_{c}$ is observable (detectable) if and only if $\tilde{\Sigma}_{d}$ is observable (detectable).

2. Effects of nonsingular state, output and input transformations, together with state feedback and output injection laws

(a) For any given nonsingular state, output and input transformations $T_{s}, T_{o}$ and $T_{i}$, the quadruple $\left(T_{s}^{-1} A T_{s}, T_{s}^{-1} B T_{i}, T_{o}^{-1} C T_{s}, T_{o}^{-1} D T_{i}\right)$ is the continuous-time counterpart of the discrete-time system $\left(T_{s}^{-1} \tilde{A} T_{s}, T_{s}^{-1} \tilde{B} T_{i}, T_{o}^{-1} \tilde{C} T_{s}, T_{o}^{-1} \tilde{D} T_{i}\right)$ under the inverse bilinear transformation (3.14).

(b) For any $\tilde{F} \in \mathbb{R}^{m \times n}$ with $\tilde{A}+\tilde{B} \tilde{F}$ having no eigenvalues at -1 , define a nonsingular matrix

$$
T_{i}:=I-\tilde{F}(I+\tilde{A}+\tilde{B} \tilde{F})^{-1} \tilde{B} \in \mathbb{R}^{m \times m}
$$

and a constant matrix

$$
F:=\sqrt{2 a} \tilde{F}(I+\tilde{A}+\tilde{B} \tilde{F})^{-1} \in \mathbb{R}^{m \times n} .
$$

Then a discrete-time system $\tilde{\Sigma}_{d \mathrm{~F}}$ characterized by $(\tilde{A}+\tilde{B} \tilde{F}, \tilde{B}, \tilde{C}+\tilde{D} \tilde{F}, \tilde{D})$ is mapped to a continuous-time counterpart $\tilde{\Sigma}_{c \mathrm{~F}}$ characterized by $\left(A+B F, B T_{i}, C+D F, D T_{i}\right)$ under the inverse bilinear transformation (3.14). 
(c) For any $\tilde{K} \in \mathbb{R}^{n \times p}$ with $\tilde{A}+\tilde{K} \tilde{C}$ having no eigenvalues at -1 , define a nonsingular matrix

$$
T_{o}:=\left[I-\tilde{C}(I+\tilde{A}+\tilde{K} \tilde{C})^{-1} \tilde{K}\right]^{-1} \in \mathbb{R}^{p \times p}
$$

and a constant matrix

$$
K:=\sqrt{2 a}(I+\tilde{A}+\tilde{K} \tilde{C})^{-1} \tilde{K} .
$$

Then a discrete-time system $\tilde{\Sigma}_{d \mathrm{~K}}$ characterized by $(\tilde{A}+\tilde{K} \tilde{C}, \tilde{B}+\tilde{K} \tilde{D}, \tilde{C}, \tilde{D})$ is mapped to a continuous-time $\tilde{\Sigma}_{c K}$, characterized by $\left(A+K C, B+K D, T_{o}^{-1} C, T_{o}^{-1} D\right)$, under the inverse bilinear transformation (3.14).

3. Invertibility and structural invariant indices lists $\mathcal{I}_{2}$ and $\mathcal{I}_{3}$ of $\tilde{\Sigma}_{c}$

(a) $\mathcal{I}_{2}\left(\tilde{\Sigma}_{c}\right)=\mathcal{I}_{2}\left(\tilde{\Sigma}_{d}\right)$, and $\mathcal{I}_{3}\left(\tilde{\Sigma}_{c}\right)=\mathcal{I}_{3}\left(\tilde{\Sigma}_{d}\right)$.

(b) $\tilde{\Sigma}_{c}$ is left (right) invertible if and only if $\tilde{\Sigma}_{d}$ is left (right) invertible.

(c) $\tilde{\Sigma}_{c}$ is invertible (degenerate) if and only if $\tilde{\Sigma}_{d}$ is invertible (degenerate).

4. Invariant zeros of $\tilde{\Sigma}_{c}$ and their structures consist of the following two parts:

(a) Let the infinite zero structure (of order greater than 0 ) of $\tilde{\Sigma}_{d}$ be given by $S_{\infty}^{\star}\left(\tilde{\Sigma}_{d}\right)=$ $\left\{q_{1}, q_{2}, \cdots, q_{m_{d}}\right\}$. Then $s=a$ is an invariant zero of $\tilde{\Sigma}_{c}$ with multiplicity structure $S_{a}^{\star}\left(\tilde{\Sigma}_{c}\right)=\left\{q_{1}, q_{2}, \cdots, q_{m_{d}}\right\}$.

(b) Let $z=\alpha \neq-1$ be an invariant zero of $\tilde{\Sigma}_{d}$ with multiplicity structure $S_{\alpha}^{\star}\left(\tilde{\Sigma}_{d}\right)=$ $\left\{n_{\alpha, 1}, n_{\alpha, 2}, \cdots, n_{\alpha, \tau_{\alpha}}\right\}$. Then $s=\beta=a(\alpha-1) /(\alpha+1)$ is an invariant zero of $\tilde{\Sigma}_{c}$ with multiplicity structure $S_{\beta}^{\star}\left(\tilde{\Sigma}_{c}\right)=\left\{n_{\alpha, 1}, n_{\alpha, 2}, \cdots, n_{\alpha, \tau_{\alpha}}\right\}$.

5. The infinite zero structure of $\tilde{\Sigma}_{c}$ consists of the following two parts:

(a) Let $m_{0}$ be the number of infinite zeros of $\tilde{\Sigma}_{d}$ of order 0 , i.e., $m_{0}=\operatorname{rank}(\tilde{D})$, and let $m_{d}$ be the total number of infinite zeros of $\tilde{\Sigma}_{d}$ of order greater than 0 . Also, let $\tau_{-1}$ be the geometric multiplicity of the invariant zero of $\tilde{\Sigma}_{d}$ at $z=-1$. Then the total number of infinite zeros of $\tilde{\Sigma}_{c}$ of order 0 , i.e., rank $(D)$, is equal to $m_{0}+m_{d}-\tau_{-1}$.

(b) Let $z=-1$ be an invariant zero of the given discrete-time system $\tilde{\Sigma}_{d}$ with multiplicity structure $S_{-1}^{\star}\left(\tilde{\Sigma}_{d}\right)=\left\{n_{-1,1}, n_{-1,2}, \cdots, n_{-1, \tau_{-1}}\right\}$. Then $\tilde{\Sigma}_{c}$ has an infinite zero (of order greater than 0) structure $S_{\infty}^{\star}\left(\tilde{\Sigma}_{c}\right)=\left\{n_{-1,1}, n_{-1,2}, \cdots, n_{-1, \tau_{-1}}\right\}$.

\section{Proof of Main Results}

We give in this section a detailed proof of Theorem 3.1 of the previous section. The derivation of Theorem 3.2 follows very similar lines to that of Theorem 3.1 and thus is omitted.

Proof of Theorem 3.1: For the sake of simplicity of presentation, and without loss of any generality, we assume that $a=2 / T=1$ throughout the proof.

1(a). Let $\beta$ be an eigenvalue of $\tilde{A}$, i.e., $\beta \in \lambda(\tilde{A})$. It is straightforward to verify that $\beta \neq-1$, provided $A$ has no eigenvalues at $a=1$ and $\alpha=(\beta-1) /(\beta+1)$ is an eigenvalue of $A$, i.e., 
$\alpha \in \lambda(A)$. Next, consider the matrix pencil

$$
\begin{aligned}
& {\left[\begin{array}{ll}
\beta I-\tilde{A} & \tilde{B}
\end{array}\right]=\left[\beta I-(I-A)^{-1}(I+A) \quad \sqrt{2}(I-A)^{-1} B\right]} \\
& =(I-A)^{-1}[\beta(I-A)-(I+A) \quad \sqrt{2} B] \\
& =(I-A)^{-2}[(\beta-1) I-(\beta+1) A \quad \sqrt{2} B] \\
& =(I-A)^{-2}\left[\begin{array}{ll}
\alpha I-A & B
\end{array}\right]\left[\begin{array}{cc}
(\beta+1) I_{n} & 0 \\
0 & \sqrt{2} I_{m}
\end{array}\right] \text {. }
\end{aligned}
$$

Clearly, $\operatorname{rank}\left[\begin{array}{lll}\beta I-\tilde{A} & \tilde{B}\end{array}\right]=\operatorname{rank}\left[\begin{array}{ll}\alpha I-A & B\end{array}\right]$, and the result $1($ a) follows.

1 (b) is the dual of $1(\mathrm{a})$ and $2(\mathrm{a})$ is trivial.

2(b). It follows from Lemma 3.1 that the discrete-time counterpart $\Sigma_{d \mathrm{~F}}$ of the bilinear transformation (with $a=1)$ of $\Sigma_{c \mathrm{~F}}$, characterized by $(A+B F, B, C+D F, D)$, is given by $\left(\tilde{A}_{\mathrm{F}}, \tilde{B}_{\mathrm{F}}, \tilde{C}_{\mathrm{F}}, \tilde{D}_{\mathrm{F}}\right)$ with

$$
\left.\begin{array}{l}
\tilde{A}_{\mathrm{F}}=(I+A+B F)(I-A-B F)^{-1} \\
\tilde{B}_{\mathrm{F}}=\sqrt{2}(I-A-B F)^{-1} B \\
\tilde{C}_{\mathrm{F}}=\sqrt{2}(C+D F)(I-A-B F)^{-1} \\
\tilde{D}_{\mathrm{F}}=D+(C+D F)(I-A-B F)^{-1} B
\end{array}\right\}
$$

We first recall from the Appendix of Kailath (1980) the following matrix identities that are frequently used in the derivation of our result:

$$
(I+X Y)^{-1} X=X(I+Y X)^{-1}
$$

and

$$
\left[I+X(s I-Z)^{-1} Y\right]^{-1}=I-X(s I-Z+X Y)^{-1} Y .
$$

Next, we note that

$$
\begin{aligned}
\tilde{A}_{\mathrm{F}} & =(I+A+B F)(I-A-B F)^{-1} \\
& =(I+A+B F)(I-A)^{-1}\left[I-B F(I-A)^{-1}\right]^{-1} \\
& =\left[\tilde{A}+B F(I-A)^{-1}\right]\left[I-B F(I-A)^{-1}\right]^{-1} \\
& =\left[\tilde{A}+B F(I-A)^{-1}\right]\left[I+B F(I-A-B F)^{-1}\right] \\
& =\tilde{A}+\tilde{A} B F(I-A-B F)^{-1}+B F(I-A)^{-1}\left[I+B F(I-A-B F)^{-1}\right] \\
& =\tilde{A}+\tilde{A} B F(I-A-B F)^{-1}+B F(I-A)^{-1}(I-A)(I-A-B F)^{-1} \\
& =\tilde{A}+\tilde{A} B F(I-A-B F)^{-1}+B F(I-A-B F)^{-1} \\
& =\tilde{A}+(\tilde{A}+I) B F(I-A-B F)^{-1} \\
& =\tilde{A}+2(I-A)^{-1} B F(I-A-B F)^{-1}=\tilde{A}+\tilde{B} \tilde{F}, \\
\tilde{B}_{\mathrm{F}} & =\sqrt{2}(I-A-B F)^{-1} B \\
& =\sqrt{2}\left[I-(I-A)^{-1} B F\right]^{-1}(I-A)^{-1} B \\
& =\sqrt{2}(I-A)^{-1} B\left[I-F(I-A)^{-1} B\right]^{-1}=\tilde{B} \tilde{T}_{i}, \\
\tilde{C}_{\mathrm{F}} & =\sqrt{2}(C+D F)(I-A-B F)^{-1} \\
& =\sqrt{2}(C+D F)(I-A)^{-1}\left[I-B F(I-A)^{-1}\right]^{-1} \\
& =\sqrt{2}(C+D F)(I-A)^{-1}\left[I+B F(I-A-B F)^{-1}\right]
\end{aligned}
$$




$$
\begin{aligned}
& =\sqrt{2} C(I-A)^{-1}+\sqrt{2} D F(I-A)^{-1}+\sqrt{2}(C+D F)(I-A)^{-1} B F(I-A-B F)^{-1} \\
& =\tilde{C}+\sqrt{2}\left[D F(I-A)^{-1}(I-A-B F)+(C+D F)(I-A)^{-1} B F\right](I-A-B F)^{-1} \\
& =\tilde{C}+\sqrt{2}\left[D F-D F(I-A)^{-1} B F+C(I-A)^{-1} B F+D F(I-A)^{-1} B F\right](I-A-B F)^{-1} \\
& =\tilde{C}+\left[D+C(I-A)^{-1} B\right] \sqrt{2} F(I-A-B F)^{-1}=\tilde{C}+\tilde{D} \tilde{F}, \\
\tilde{D}_{\mathrm{F}} & =D+(C+D F)(I-A-B F)^{-1} B \\
& =D+(C+D F)\left[I-(I-A)^{-1} B F\right]^{-1}(I-A)^{-1} B \\
& =D+(C+D F)(I-A)^{-1} B\left[I-F(I-A)^{-1} B\right]^{-1} \\
& =\left\{D\left[I-F(I-A)^{-1} B\right]+(C+D F)(I-A)^{-1} B\right\} \tilde{T}_{i} \\
& =\left\{D-D F(I-A)^{-1} B+C(I-A)^{-1} B+D F(I-A)^{-1} B\right\} \tilde{T}_{i}=\tilde{D} \tilde{T}_{i},
\end{aligned}
$$

which completes the proof of $2(\mathrm{~b})$.

2(c). Dual of $2(\mathrm{~b})$.

With the benefit of properties of $2(\mathrm{a})-2(\mathrm{c})$, the remainder of the proof is considerably simplified. It is well known that the structural invariant indices lists of Morse, which correspond precisely to the structures of finite and infinite zeros as well as invertibility, are invariant under nonsingular state, output and input transformations, state feedback laws and output injections. We can thus apply appropriate nonsingular state, output and input transformations, as well as state feedback and output injection, to $\Sigma_{c}$ and so obtain a new system, say $\Sigma_{c}^{*}$. If this new system has $\Sigma_{d}^{*}$ as its discrete-time counterpart under bilinear transformation, then from properties 2(a)-2(c), it follows that $\Sigma_{d}^{*}$ and $\Sigma_{d}$ have the same structural invariant properties. It is therefore sufficient for the remainder of the proof that we show $3(\mathrm{a})-5(\mathrm{~b})$ are properties of $\Sigma_{d}^{*}$.

Let us first apply nonsingular state, output and input transformations $\Gamma_{s}, \Gamma_{o}$ and $\Gamma_{i}$ to $\Sigma_{c}$ such that the resulting system is in the form of the special coordinate basis as in Theorem 2.1, or, equivalently, the compact form in (2.12)-(2.14). We will assume that $A_{a a}$ is already in the Jordan form of (2.15) and (2.16), and that matrices $A_{a a}, L_{a d}, B_{a 0}, E_{d a}, C_{0 a}, E_{c a}$ and $L_{a b}$ are partitioned as follows:

$$
\begin{gathered}
A_{a a}=\left[\begin{array}{cc}
A_{a a}^{a} & 0 \\
0 & A_{a a}^{*}
\end{array}\right], \quad L_{a d}=\left[\begin{array}{c}
L_{a d}^{a} \\
L_{a d}^{*}
\end{array}\right], \quad B_{a 0}=\left[\begin{array}{c}
B_{a 0}^{a} \\
B_{a 0}^{*}
\end{array}\right], \quad L_{a b}=\left[\begin{array}{c}
L_{a b}^{a} \\
L_{a b}^{*}
\end{array}\right] \\
E_{d a}=\left[\begin{array}{ll}
E_{d a}^{a} & E_{d a}^{*}
\end{array}\right], \quad C_{0 a}=\left[\begin{array}{ll}
C_{0 a}^{a} & C_{0 a}^{*}
\end{array}\right], \quad E_{c a}=\left[\begin{array}{ll}
E_{c a}^{a} & E_{c a}^{*}
\end{array}\right] .
\end{gathered}
$$

Moreover, since $A_{a a}^{a}$ has all its eigenvalues at $a=1$,

$$
A_{a a}^{a}=I+\left[\begin{array}{ccccc}
0 & I_{n_{a, 1}-1} & \cdots & 0 & 0 \\
0 & 0 & \cdots & 0 & 0 \\
\vdots & \vdots & \ddots & \vdots & \vdots \\
0 & 0 & \cdots & 0 & I_{n_{a, \tau_{a}}-1} \\
0 & 0 & \cdots & 0 & 0
\end{array}\right]
$$

while $A_{a a}^{*}$ contains the remaining invariant zeros of $\Sigma_{c}$. Furthermore, we assume that the pair $\left(A_{c c}, B_{c}\right)$ is in the Brunovsky canonical form of $(2.19)$, as is the pair $\left(A_{b b}^{\prime}, C_{b}^{\prime}\right)$. Next, define a 
state feedback gain matrix

$$
F=-\Gamma_{i}\left[\begin{array}{ccccc}
C_{0 a}^{a}-C_{2}^{a} & C_{0 a}^{*} & C_{0 b} & C_{0 c} & C_{0 d} \\
E_{d a}^{a}-C_{1}^{a} & E_{d a}^{*} & E_{d b} & E_{d c} & E_{d d} \\
E_{c a}^{a} & E_{c a}^{*} & 0 & E_{c c} & 0
\end{array}\right] \Gamma_{s}^{-1}
$$

and an output injection gain matrix

$$
K=-\Gamma_{s}\left[\begin{array}{ccc}
B_{a 0}^{a}-B_{2}^{a} & L_{a d}^{a}-B_{1}^{a} & L_{a b}^{a} \\
B_{a 0}^{*} & L_{a d}^{*} & L_{a b}^{*} \\
B_{b 0} & L_{b d} & L_{b b} \\
B_{c 0} & L_{c d} & L_{c b} \\
B_{d 0} & L_{d d} & 0
\end{array}\right] \Gamma_{o}^{-1}
$$

Here, $E_{c c}$ is chosen such that all $\star$ 's in (2.19) are cleaned out, i.e., $A_{c c}^{*}:=A_{c c}-B_{c} E_{c c}$ is in Jordan form with all diagonal elements equal to 0. Similarly, $L_{b b}$ is chosen such that $\left(A_{b b}^{*}\right)^{\prime}:=$ $\left(A_{b b}-L_{b b} C_{b}\right)^{\prime}$ is in Jordan form with with all diagonal elements equal to 0 . Likewise, $E_{d d}$ and $L_{d d}$ are chosen such that $A_{d d}^{*}:=A_{d d}-L_{d d} C_{d}-B_{d} E_{d d}$ is in Jordan form with all diagonal elements equal to 0 , which in turn implies

$$
C_{d}\left(I-A_{d d}^{*}\right)^{-1} B_{d}=I_{m_{d}}
$$

The matrices $B_{1}^{a}, B_{2}^{a}, C_{1}^{a}$ and $C_{2}^{a}$ are chosen in conformity with $A_{a a}^{a}$ of (4.6) as follows:

$$
B^{a}:=\left[\begin{array}{ll}
B_{2}^{a} & B_{1}^{a}
\end{array}\right]:=\left[\begin{array}{cccc}
0 & 0 & \cdots & 0 \\
0 & 1 & \cdots & 0 \\
\vdots & \ddots & \vdots & \vdots \\
0 & 0 & \cdots & 0 \\
0 & 0 & \cdots & 1
\end{array}\right], \quad C^{a}:=\left[\begin{array}{c}
C_{2}^{a} \\
C_{1}^{a}
\end{array}\right]:=\left[\begin{array}{ccccc}
0 & 0 & \cdots & 0 & 0 \\
1 & 0 & \cdots & 0 & 0 \\
\vdots & \vdots & \ddots & \vdots & \vdots \\
0 & 0 & \cdots & 1 & 0
\end{array}\right] .
$$

This can always be done, as a consequence of the assumption that the matrix $A$ has no eigenvalues at $a=1$, which implies that the invariant zero at $a=1$ of $\Sigma_{c}$ is completely controllable and observable.

Finally, we obtain a continuous-time system $\Sigma_{c}^{*}$ characterized by the quadruple $\left(A^{*}, B^{*}, C^{*}, D^{*}\right)$, where

$$
\begin{gathered}
A^{*}=P^{-1} \Gamma_{s}^{-1}(A+B F+K C+K D F) \Gamma_{s} P=\left[\begin{array}{ccccc}
A_{a a}^{*} & 0 & 0 & 0 & 0 \\
0 & A_{b b}^{*} & 0 & 0 & 0 \\
0 & 0 & A_{c c}^{*} & 0 & 0 \\
0 & 0 & 0 & A_{d d}^{*} & B_{d} C_{1}^{a} \\
0 & 0 & 0 & B_{1}^{a} C_{d} & A_{a a}^{a}+B_{2}^{a} C_{2}^{a}
\end{array}\right] \\
B^{*}=P^{-1} \Gamma_{s}^{-1}(B+K D) \Gamma_{i}=\left[\begin{array}{ccc}
0 & 0 & 0 \\
0 & 0 & 0 \\
0 & 0 & B_{c} \\
0 & B_{d} & 0 \\
B_{2}^{a} & 0 & 0
\end{array}\right]
\end{gathered}
$$

and

$$
C^{*}=\Gamma_{o}^{-1}(C+D F) \Gamma_{s} P=\left[\begin{array}{ccccc}
0 & 0 & 0 & 0 & C_{2}^{a} \\
0 & 0 & 0 & C_{d} & 0 \\
0 & C_{b} & 0 & 0 & 0
\end{array}\right], \quad D^{*}=\Gamma_{o}^{-1} D \Gamma_{i}=\left[\begin{array}{ccc}
I_{m_{0}} & 0 & 0 \\
0 & 0 & 0 \\
0 & 0 & 0
\end{array}\right],
$$


where $P$ is a permutation matrix that transforms $A_{a a}^{a}$ from its original position, i.e., block $(1,1)$, to block $(5,5)$ in $(4.11)$.

Next, define a subsystem $\left(A_{s}, B_{s}, C_{s}, D_{s}\right)$ with

$$
A_{s}:=\left[\begin{array}{cc}
A_{d d}^{*} & B_{d} C_{1}^{a} \\
B_{1}^{a} C_{d} & A_{a a}^{a}+B_{2}^{a} C_{2}^{a}
\end{array}\right], \quad B_{s}:=\left[\begin{array}{cc}
0 & B_{d} \\
B_{2}^{a} & 0
\end{array}\right]
$$

and

$$
C_{s}:=\left[\begin{array}{cc}
0 & C_{2}^{a} \\
C_{d} & 0
\end{array}\right], \quad D_{s}:=\left[\begin{array}{cc}
I_{m_{0}} & 0 \\
0 & 0
\end{array}\right] .
$$

It is straightforward to verify that with the choice of $B^{a}$ and $C^{a}$ as in (4.10), $A_{s}$ has no eigenvalues at $a=1$. Hence $A^{*}$ has no eigenvalues at $a=1$ either, since both $A_{b b}^{*}$ and $A_{c c}^{*}$ have all eigenvalues at 0 , and $A_{a a}^{*}$ contains only the invariant zeros of $\Sigma_{c}$ which are not equal to $a=1$. Applying the bilinear transformation (3.3) to $\Sigma_{c}^{*}$, it follows from Lemma 3.1 that we obtain a discrete-time system $\Sigma_{d}^{*}$, characterized by $\left(\tilde{A}^{*}, \tilde{B}^{*}, \tilde{C}^{*}, \tilde{D}^{*}\right)$, with

$$
\begin{gathered}
\tilde{A}^{*}=\left[\begin{array}{cccc}
\left(I+A_{a a}^{*}\right)\left(I-A_{a a}^{*}\right)^{-1} & 0 & 0 & 0 \\
0 & \left(I+A_{b b}^{*}\right)\left(I-A_{b b}^{*}\right)^{-1} & 0 & 0 \\
0 & 0 & \left(I+A_{c c}^{*}\right)\left(I-A_{c c}^{*}\right)^{-1} & 0 \\
0 & 0 & 0 \\
0 & \tilde{B}^{*}=\sqrt{2}\left[\begin{array}{cc}
0 \\
0
\end{array}\right], \\
0 & \left(I-A_{s c}^{*}\right)^{-1} B_{c} \\
\left(I-A_{s}\right)^{-1} B_{s} & 0
\end{array}\right],
\end{gathered}
$$

and

$$
\tilde{C}^{*}=\sqrt{2}\left[\begin{array}{cccc}
0 & 0 & 0 & C_{s}\left(I-A_{s}\right)^{-1} \\
0 & C_{b}\left(I-A_{s}\right)^{-1} & 0 & 0
\end{array}\right], \quad \tilde{D}^{*}=\left[\begin{array}{cc}
D_{s}+C_{s}\left(I-A_{s}\right)^{-1} B_{s} & 0 \\
0 & 0
\end{array}\right] .
$$

Our next task is to find appropriate transformations, state feedback, and output injection laws, so as to transform the above system into the form of the special coordinate basis displaying the properties $3(\mathrm{a})-5(\mathrm{~b})$.

To simplify the presentation, we first focus on the subsystem $\left(\tilde{A}_{s}, \tilde{B}_{s}, \tilde{C}_{s}, \tilde{D}_{s}\right)$ with

$$
\tilde{A}_{s}:=\left(I+A_{s}\right)\left(I-A_{s}\right)^{-1}, \quad \tilde{B}_{s}:=\sqrt{2}\left(I-A_{s}\right)^{-1} B_{s}
$$

and

$$
\tilde{C}_{s}:=\sqrt{2} C_{s}\left(I-A_{s}\right)^{-1}, \quad \tilde{D}_{s}:=D_{s}+C_{s}\left(I-A_{s}\right)^{-1} B_{s} .
$$

Using (4.9) in conjunction with Appendix A.22 of Kailath (1980), it is straightforward to compute

$$
\tilde{A}_{s}=\left[\begin{array}{cc}
X_{2} & 2\left(I-A_{d d}^{*}\right)^{-1} B_{d} C_{1}^{a}\left(I-A_{a a}^{a}-B^{a} C^{a}\right)^{-1} \\
2\left(I-A_{a a}^{a}-B^{a} C^{a}\right)^{-1} B_{1}^{a} C_{d}\left(I-A_{d d}^{*}\right)^{-1} & \left(I+A_{a a}^{a}+B^{a} C^{a}\right)\left(I-A_{a a}^{a}-B^{a} C^{a}\right)^{-1}
\end{array}\right],
$$

where

$$
\begin{gathered}
X_{2}=\left(I+A_{d d}^{*}\right)\left(I-A_{d d}^{*}\right)^{-1}+2\left(I-A_{d d}^{*}\right)^{-1} B_{d} C_{1}^{a}\left(I-A_{a a}^{a}-B^{a} C^{a}\right)^{-1} B_{1}^{a} C_{d}\left(I-A_{d d}^{*}\right)^{-1}, \\
\tilde{B}_{s}=\sqrt{2}\left[\begin{array}{cc}
\left(I-A_{d d}^{*}\right)^{-1} B_{d} C_{1}^{a}\left(I-A_{a a}^{a}-B^{a} C^{a}\right)^{-1} B_{2}^{a} & \left(I-A_{d d}^{*}\right)^{-1} B_{d}\left[I+C_{1}^{a}\left(I-A_{a a}^{a}-B^{a} C^{a}\right)^{-1} B_{1}^{a}\right] \\
\left(I-A_{a a}^{a}-B^{a} C^{a}\right)^{-1} B_{2}^{a} & \left(I-A_{a a}^{a}-B^{a} C^{a}\right)^{-1} B_{1}^{a}
\end{array}\right]
\end{gathered}
$$




$$
\tilde{C}_{s}=\sqrt{2}\left[\begin{array}{cc}
C_{2}^{a}\left(I-A_{a a}^{a}-B^{a} C^{a}\right)^{-1} B_{1}^{a} C_{d}\left(I-A_{d d}^{*}\right)^{-1} & C_{2}^{a}\left(I-A_{a a}^{a}-B^{a} C^{a}\right)^{-1} \\
{\left[I+C_{1}^{a}\left(I-A_{a a}^{a}-B^{a} C^{a}\right)^{-1} B_{1}^{a}\right] C_{d}\left(I-A_{d d}^{*}\right)^{-1}} & C_{1}^{a}\left(I-A_{a a}^{a}-B^{a} C^{a}\right)^{-1}
\end{array}\right]
$$

and

$$
\tilde{D}_{s}=\left[\begin{array}{cc}
I+C_{2}^{a}\left(I-A_{a a}^{a}-B^{a} C^{a}\right)^{-1} B_{2}^{a} & C_{2}^{a}\left(I-A_{a a}^{a}-B^{a} C^{a}\right)^{-1} B_{1}^{a} \\
C_{1}^{a}\left(I-A_{a a}^{a}-B^{a} C^{a}\right)^{-1} B_{2}^{a} & I+C_{1}^{a}\left(I-A_{a a}^{a}-B^{a} C^{a}\right)^{-1} B_{1}^{a}
\end{array}\right] .
$$

Noting the structure of $A_{a a}^{a}$ in (4.6), and the structures of $B^{a}$ and $C^{a}$ in (4.10), we have

$$
\begin{gathered}
\left(I-A_{a a}-B^{a} C^{a}\right)^{-1}=\left[\begin{array}{ccccc}
0 & -1 & \cdots & 0 & 0 \\
-I_{n_{a, 1}-1} & 0 & \cdots & 0 & 0 \\
\vdots & \vdots & \ddots & \vdots & \vdots \\
0 & 0 & \cdots & 0 & -1 \\
0 & 0 & \cdots & -I_{n_{a, \tau_{a}}-1} & 0
\end{array}\right], \\
C_{1}^{a}\left(I-A_{a a}-B^{a} C^{a}\right)^{-1} B_{2}^{a}=0, \quad C_{2}^{a}\left(I-A_{a a}-B^{a} C^{a}\right)^{-1} B_{1}^{a}=0
\end{gathered}
$$

and

$$
C^{a}\left(I-A_{a a}-B^{a} C^{a}\right)^{-1} B^{a}=\left[\begin{array}{cc}
0 & 0 \\
0 & -I_{\tau_{a}}
\end{array}\right] .
$$

Thus, $\tilde{B}_{s}, \tilde{C}_{s}$ and $\tilde{D}_{s}$ are reduced to the following forms:

$$
\begin{aligned}
& \tilde{B}_{s}=\sqrt{2}\left[\begin{array}{cc}
0 & \left(I-A_{d d}^{*}\right)^{-1} B_{d}\left[I+C_{1}^{a}\left(I-A_{a a}^{a}-B^{a} C^{a}\right)^{-1} B_{1}^{a}\right] \\
\left(I-A_{a a}^{a}-B^{a} C^{a}\right)^{-1} B_{2}^{a} & \left(I-A_{a a}^{a}-B^{a} C^{a}\right)^{-1} B_{1}^{a}
\end{array}\right], \\
& \tilde{C}_{s}=\sqrt{2}\left[\begin{array}{cc}
0 & C_{2}^{a}\left(I-A_{a a}^{a}-B^{a} C^{a}\right)^{-1} \\
{\left[I+C_{1}^{a}\left(I-A_{a a}^{a}-B^{a} C^{a}\right)^{-1} B_{1}^{a}\right] C_{d}\left(I-A_{d d}^{*}\right)^{-1}} & C_{1}^{a}\left(I-A_{a a}^{a}-B^{a} C^{a}\right)^{-1}
\end{array}\right]
\end{aligned}
$$

and

$$
\tilde{D}_{s}=\left[\begin{array}{cc}
I+C_{2}^{a}\left(I-A_{a a}^{a}-B^{a} C^{a}\right)^{-1} B_{2}^{a} & 0 \\
0 & I+C_{1}^{a}\left(I-A_{a a}^{a}-B^{a} C^{a}\right)^{-1} B_{1}^{a}
\end{array}\right]
$$

Next, define

$$
\tilde{F}_{s}:=\sqrt{2}\left[\begin{array}{cc}
0 & 0 \\
-C_{d}\left(I-A_{d d}^{*}\right)^{-1} & 0
\end{array}\right]
$$

and

$$
\tilde{K}_{s}:=\sqrt{2}\left[\begin{array}{cc}
0 & -\left(I-A_{d d}^{*}\right)^{-1} B_{d} \\
0 & 0
\end{array}\right]
$$

from which it follows that

$$
\tilde{A}_{s c}=\tilde{A}_{s}+\tilde{B}_{s} \tilde{F}_{s}+\tilde{K}_{s} \tilde{C}_{s}+\tilde{K}_{s} \tilde{D}_{s} \tilde{F}_{s}=\left[\begin{array}{cc}
\tilde{A}_{a a}^{* *} & 0 \\
0 & \left(I+A_{a a}^{a}+B^{a} C^{a}\right)\left(I-A_{a a}^{a}-B^{a} C^{a}\right)^{-1}
\end{array}\right],
$$

where

$$
\begin{gathered}
\tilde{A}_{a a}^{* *}:=\left(I+A_{d d}^{*}\right)\left(I-A_{d d}^{*}\right)^{-1}-2\left(I-A_{d d}^{*}\right)^{-1} B_{d} C_{d}\left(I-A_{d d}^{*}\right)^{-1}, \\
\tilde{B}_{s c}=\tilde{B}_{s}+\tilde{K}_{s} \tilde{D}_{s}=\sqrt{2}\left[\begin{array}{cc}
0 & 0 \\
\left(I-A_{a a}^{a}-B^{a} C^{a}\right)^{-1} B_{2}^{a} & \left(I-A_{a a}^{a}-B^{a} C^{a}\right)^{-1} B_{1}^{a}
\end{array}\right],
\end{gathered}
$$

and

$$
\tilde{C}_{s c}=\tilde{C}_{s}+\tilde{D}_{s} \tilde{F}_{s}=\sqrt{2}\left[\begin{array}{cc}
0 & C_{2}^{a}\left(I-A_{a a}^{a}-B^{a} C^{a}\right)^{-1} \\
0 & C_{1}^{a}\left(I-A_{a a}^{a}-B^{a} C^{a}\right)^{-1}
\end{array}\right] .
$$

Next, repartition $B^{a}$ and $C^{a}$ of (4.10) as follows:

$$
B^{a}=\left[\begin{array}{ll}
0 & \tilde{B}_{a}
\end{array}\right] \quad \text { and } \quad C^{a}=\left[\begin{array}{c}
0 \\
\tilde{C}_{a}
\end{array}\right],
$$


where both $\tilde{B}_{a}$ and $\tilde{C}_{a}$ are of maximal rank. We thus obtain

$$
\begin{gathered}
\tilde{A}_{s c}=\left[\begin{array}{cc}
\tilde{A}_{a a}^{* *} & 0 \\
0 & \left(I+A_{a a}^{a}+\tilde{B}_{a} \tilde{C}_{a}\right)\left(I-A_{a a}^{a}-\tilde{B}_{a} \tilde{C}_{a}\right)^{-1}
\end{array}\right], \\
\tilde{B}_{s c}=\sqrt{2}\left[\begin{array}{cc}
0 & 0 \\
0 & \left(I-A_{a a}-\tilde{B}_{a} \tilde{C}_{a}\right)^{-1} \tilde{B}_{a}
\end{array}\right],
\end{gathered}
$$

and

$$
\tilde{C}_{s c}=\sqrt{2}\left[\begin{array}{cc}
0 & 0 \\
0 & \tilde{C}_{a}\left(I-A_{a a}-\tilde{B}_{a} \tilde{C}_{a}\right)^{-1}
\end{array}\right], \quad \tilde{D}_{s c}=\tilde{D}_{s}=\left[\begin{array}{cc}
I_{m_{0}+m_{d}-\tau_{a}} & 0 \\
0 & 0
\end{array}\right] .
$$

Using (4.6) and (4.25), straightforward manipulations yield

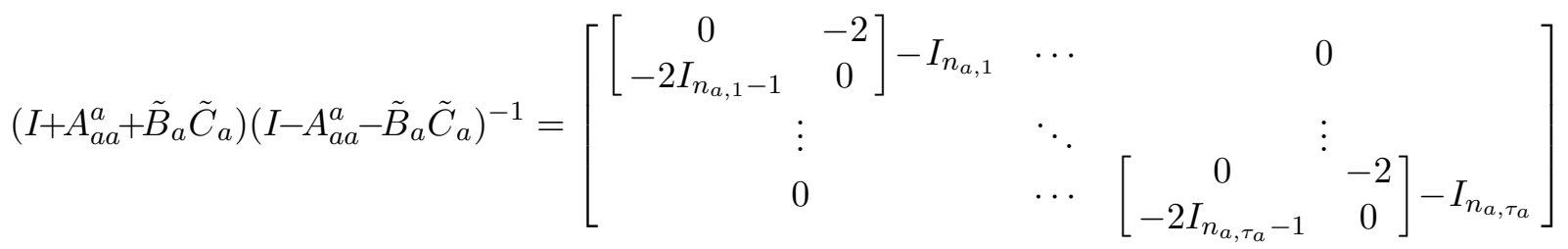

$$
\begin{aligned}
& \left(I-A_{a a}^{a}-\tilde{B}_{a} \tilde{C}_{a}\right)^{-1} \tilde{B}_{a}=-\left[\begin{array}{ccc}
1 & \cdots & 0 \\
0 & \cdots & 0 \\
\vdots & \ddots & \vdots \\
0 & \cdots & 1 \\
0 & \cdots & 0
\end{array}\right]
\end{aligned}
$$

and

$$
\tilde{C}_{a}\left(I-A_{a a}^{a}-\tilde{B}_{a} \tilde{C}_{a}\right)^{-1}=-\left[\begin{array}{ccccc}
0 & 1 & \cdots & 0 & 0 \\
\vdots & \vdots & \ddots & \vdots & \vdots \\
0 & 0 & \cdots & 0 & 1
\end{array}\right] .
$$

Moreover, it can be readily verified that each subsystem $\left(\tilde{A}_{a i}, \tilde{B}_{a i}, \tilde{C}_{a i}\right), i=1, \cdots, \tau_{a}$, with

$$
\tilde{A}_{a i}=-I_{n_{a, i}}+\left[\begin{array}{cc}
0 & -2 \\
-2 I_{n_{a, i}-1} & 0
\end{array}\right], \quad \tilde{B}_{a i}=\left[\begin{array}{r}
-1 \\
0
\end{array}\right], \quad \tilde{C}_{a i}=\left[\begin{array}{ll}
0 & -1
\end{array}\right],
$$

has the following properties:

$$
\tilde{C}_{a i} \tilde{B}_{a i}=\tilde{C}_{a i} \tilde{A}_{a i} \tilde{B}_{a i}=\cdots=\tilde{C}_{a i}\left(\tilde{A}_{a i}\right)^{n_{a, i}-2} \tilde{B}_{a i}=0 \quad \text { and } \quad \tilde{C}_{a i}\left(\tilde{A}_{a i}\right)^{n_{a, i}-1} \tilde{B}_{a i} \neq 0 .
$$

It follows from Theorem 2.1 that there exist nonsingular transformations $\Gamma_{s a}, \Gamma_{o a}$ and $\Gamma_{i a}$ such that

$$
\begin{gathered}
\tilde{A}_{d}=\Gamma_{s a}^{-1}\left[\left(I+A_{a a}^{a}+\tilde{B}_{a} \tilde{C}_{a}\right)\left(I-A_{a a}^{a}-\tilde{B}_{a} \tilde{C}_{a}\right)^{-1}\right] \Gamma_{s a}=\left[\begin{array}{ccccc}
\star & I_{n_{a, 1}-1} & \cdots & 0 & 0 \\
\star & \star & \cdots & 0 & 0 \\
\vdots & \vdots & \ddots & \vdots & \vdots \\
0 & 0 & \cdots & \star & I_{n_{a, \tau_{a}-1}} \\
0 & 0 & \cdots & \star & \star
\end{array}\right], \\
\tilde{B}_{d}=\Gamma_{s a}^{-1}\left[\left(I-A_{a a}^{a}-\tilde{B}_{a} \tilde{C}_{a}\right)^{-1} \tilde{B}_{a}\right] \Gamma_{i a}=\left[\begin{array}{ccc}
0 & \cdots & 0 \\
1 & \cdots & 0 \\
\vdots & \ddots & \vdots \\
0 & \cdots & 0 \\
0 & \cdots & 1
\end{array}\right],
\end{gathered}
$$


and

$$
\tilde{C}_{d}=\Gamma_{o a}^{-1}\left[\tilde{C}_{a}\left(I-A_{a a}^{a}-\tilde{B}_{a} \tilde{C}_{a}\right)^{-1}\right] \Gamma_{s a}=\left[\begin{array}{ccccc}
1 & 0 & \cdots & 0 & 0 \\
\vdots & \vdots & \ddots & \vdots & \vdots \\
0 & 0 & \cdots & 1 & 0
\end{array}\right]
$$

Now, let us return to $\Sigma_{d}^{*}$ characterized by $\left(\tilde{A}^{*}, \tilde{B}^{*}, \tilde{C}^{*}, \tilde{D}^{*}\right)$ as in (4.16) to (4.18). Using the properties of the subsystem $\left(\tilde{A}_{s}, \tilde{B}_{s}, \tilde{C}_{s}, \tilde{D}_{s}\right)$ just derived, we are in a position to define appropriate state feedback and output injection gain matrices, say $\tilde{F}^{*}$ and $\tilde{K}^{*}$, together with nonsingular state, output and input transformations $\tilde{\Gamma}_{s}^{*}, \tilde{\Gamma}_{o}^{*}$ and $\tilde{\Gamma}_{i}^{*}$, such that

$$
\begin{aligned}
& \tilde{A}_{\mathrm{SCB}}^{*}:=\left(\tilde{\Gamma}_{s}^{*}\right)^{-1}\left(\tilde{A}^{*}+\tilde{B}^{*} \tilde{F}^{*}+\tilde{K}^{*} \tilde{C}^{*}+\tilde{K}^{*} \tilde{D}^{*} \tilde{F}^{*}\right) \tilde{\Gamma}_{s}^{*} \\
& =\left[\begin{array}{ccccc}
\left(I+A_{a a}^{*}\right)\left(I-A_{a a}^{*}\right)^{-1} & 0 & 0 & 0 & 0 \\
0 & \left(I+A_{b b}^{*}\right)\left(I-A_{b b}^{*}\right)^{-1} & 0 & 0 & 0 \\
0 & 0 & \left(I+A_{c c}^{*}\right)\left(I-A_{c c}^{*}\right)^{-1} & 0 & 0 \\
0 & 0 & 0 & \tilde{A}_{a a}^{* *} & 0 \\
0 & 0 & 0 & 0 & \tilde{A}_{d}
\end{array}\right]
\end{aligned}
$$

with $\tilde{A}_{a a}^{* *}$ given by $(4.33)$, and

$$
\begin{gathered}
\tilde{B}_{\mathrm{SCB}}^{*}:=\left(\tilde{\Gamma}_{s}^{*}\right)^{-1}\left(\tilde{B}^{*}+\tilde{K}^{*} \tilde{D}^{*}\right) \tilde{\Gamma}_{i}^{*}=\left[\begin{array}{ccc}
0 & 0 & 0 \\
0 & 0 & 0 \\
0 & 0 & \left(I-A_{c c}^{*}\right)^{-1} B_{c} \\
0 & 0 & 0 \\
0 & \tilde{B}_{d} & 0
\end{array}\right], \\
\tilde{C}_{\mathrm{SCB}}^{*}:=\left(\tilde{\Gamma}_{o}^{*}\right)^{-1}\left(\tilde{C}^{*}+\tilde{D}^{*} \tilde{F}^{*}\right) \tilde{\Gamma}_{s}^{*}=\left[\begin{array}{ccccc}
0 & 0 & 0 & 0 & 0 \\
0 & C_{b}\left(I-A_{b b}^{*}\right)^{-1} & 0 & 0 & 0 \\
0 & 0 & 0 & 0 & \tilde{C}_{d}
\end{array}\right],
\end{gathered}
$$

and

$$
\tilde{D}_{\mathrm{SCB}}^{*}:=\left(\tilde{\Gamma}_{o}^{*}\right)^{-1} \tilde{D}^{*} \tilde{\Gamma}_{i}^{*}=\left[\begin{array}{ccc}
I_{m_{0}+m_{d}-\tau_{a}} & 0 & 0 \\
0 & 0 & 0 \\
0 & 0 & 0
\end{array}\right]
$$

Clearly, $\Sigma_{\mathrm{SCB}}^{*}$ characterized by $\left(\tilde{A}_{\mathrm{SCB}}^{*}, \tilde{B}_{\mathrm{SCB}}^{*}, \tilde{C}_{\mathrm{SCB}}^{*}, \tilde{D}_{\mathrm{SCB}}^{*}\right)$ has the same structural invariant indices lists as does $\Sigma_{d}^{*}$, which in turn has the same structural invariant indices lists as $\Sigma_{d}$. Most importantly, however, $\Sigma_{\mathrm{SCB}}^{*}$ is in the form of the special coordinate basis, and we are now ready to prove properties $3(\mathrm{a})-5(\mathrm{~b})$ of the theorem.

3(a). First, we note that $\mathcal{I}_{2}\left(\Sigma_{d}\right)=\mathcal{I}_{2}\left(\Sigma_{\mathrm{SCB}}^{*}\right)$. From (4.38) to (4.41) and the properties of the special coordinate basis, we know that $\mathcal{I}_{2}\left(\Sigma_{\mathrm{SCB}}^{*}\right)$ is given by the controllability index of the pair

$$
\left\{\left(I+A_{c c}^{*}\right)\left(I-A_{c c}^{*}\right)^{-1},\left(I-A_{c c}^{*}\right)^{-1} B_{c}\right\} \quad \text { or } \quad\left\{\left(I+A_{c c}^{*}\right)\left(I-A_{c c}^{*}\right)^{-1}, B_{c}\right\} .
$$

It is straightforward to verify that the controllability index of $\left\{\left(I+A_{c c}^{*}\right)\left(I-A_{c c}^{*}\right)^{-1}, B_{c}\right\}$ is the same as that of $\left(A_{c c}^{*}, B_{c}\right)$, and thus $\mathcal{I}_{2}\left(\Sigma_{d}\right)=\mathcal{I}_{2}\left(\Sigma_{c}\right)$. Likewise, the proof that $\mathcal{I}_{3}\left(\Sigma_{d}\right)=\mathcal{I}_{3}\left(\Sigma_{c}\right)$ follows along similar lines.

3(b)-3(c). Follow directly from 3(a). 
4(a). It follows from the properties of the special coordinate basis that the invariant zero structure of $\tilde{\Sigma}_{\mathrm{SCB}}^{*}$, or equivalently $\Sigma_{d}$, is given by the eigenvalues of $\tilde{A}_{a a}^{* *}$ and $\left(I+A_{a a}^{*}\right)\left(I-A_{a a}^{*}\right)^{-1}$, together with their associated Jordan blocks. Property $4(\mathrm{a})$ corresponds with the eigenvalues of $\tilde{A}_{a a}^{* *}$ of (4.33), together with their associated Jordan blocks. First, we note that for any $z \in \mathbb{C}$,

$$
z I-\tilde{A}_{a a}^{* *}=\left[(z-1) I-(z+1) A_{d d}^{*}+2\left(I-A_{d d}^{*}\right)^{-1} B_{d} C_{d}\right]\left(I-A_{d d}^{*}\right)^{-1} .
$$

It can be shown that

$$
(z-1) I-(z+1) A_{d d}^{*}+2\left(I-A_{d d}^{*}\right)^{-1} B_{d} C_{d}=\text { Block diag }\left\{Q_{1}(z), \cdots, Q_{i}(z)\right\},
$$

where $Q_{i}(z) \in \mathbb{C}^{n_{q_{i}} \times n_{q_{i}}}$ is given by

$$
Q_{i}(z)=\left[\begin{array}{cccccc}
z+1 & -(z+1) & 0 & \cdots & 0 & 0 \\
2 & z-1 & -(z+1) & \cdots & 0 & 0 \\
2 & 0 & z-1 & \ddots & 0 & 0 \\
\vdots & \vdots & \vdots & \ddots & \ddots & \vdots \\
2 & 0 & 0 & \cdots & z-1 & -(z+1) \\
2 & 0 & 0 & \cdots & 0 & z-1
\end{array}\right]
$$

for $i=1, \cdots, m_{d}$. It follows from (4.42) that the eigenvalue of $\tilde{A}_{a a}^{* *}$ is the scalar $z$ that causes the rank of Block diag $\left\{Q_{1}(z), \cdots, Q_{m_{d}}(z)\right\}$ to drop below $n_{d}=\sum_{i=1}^{m_{d}} q_{i}$. Using the particular form of $Q_{i}(z)$, it is clear that the only such scalar $z \in \mathbb{C}$ which causes $Q_{i}(z)$ to drop rank is $z=-1$. Moreover, rank $\left\{Q_{i}(-1)\right\}=n_{q_{i}}-1$, i.e., $Q_{i}(-1)$ has only one linearly independent eigenvector. Hence, $z=-1$ is the eigenvalue of $\tilde{A}_{a a}^{* *}$, or equivalently the invariant zero of $\Sigma_{d}$, with multiplicity structure $S_{-1}^{\star}\left(\Sigma_{d}\right)=\left\{q_{1}, \cdots, q_{m_{d}}\right\}=S_{\infty}^{\star}\left(\Sigma_{c}\right)$, thereby proving $4($ a) .

4(b). This part of the infinite zero structure corresponds to the invariant zeros of the matrix $\left(I+A_{a a}^{*}\right)\left(I-A_{a a}^{*}\right)^{-1}$. With $A_{a a}^{*}$ in Jordan form, Property 4(b) follows by straightforward manipulations.

5(a) follows directly from (4.41) and 5(b) follows from the structure of $\left(\tilde{A}_{d}, \tilde{B}_{d}, \tilde{C}_{d}\right)$ in (4.35) to (4.37), in conjunction with Property 2.2 of the special coordinate basis.

This concludes the proof of Theorem 3.1.

\section{Conclusion}

We have presented in this paper a comprehensive study of the structural mappings, and invertibility properties, associated with the bilinear transformation of general linear time-invariant multivariable systems. It has been demonstrated that, in general, the structural invariant indices lists $\mathcal{I}_{1}$ and $\mathcal{I}_{4}$ of Morse (1973) are changed under bilinear transformation, while the structural invariant indices lists $\mathcal{I}_{2}$ and $\mathcal{I}_{3}$ of Morse are preserved. Finally, it is worth pointing out that the Jordan canonical form, the Brunovsky canonical form, and the Special Coordinate Basis of Sannuti and Saberi, are truly remarkable tools that can be utilized to characterize all structural properties of interest for general linear time-invariant multivariable systems, as demonstrated 
in the proof of the main results. Future research will investigate the applicability of these same tools to the analysis of the structural properties of sampled-data systems generated by zero-order hold $(\mathrm{ZOH})$ sampling.

\section{References}

[1] Åström, K. J., P. Hagander, and J. Sternby (1984). Zeros of sampled systems. Automatica, Vol. 20, No. 4, pp. 21-38.

[2] Chen, B. M. (1988). Software Manual for the Special Coordinate Basis of Multivariable Linear Systems. Washington State University Technical Report Number: ECE 0094, Pullman, Washington.

[3] Chen, B. M., A. Saberi and Y. Shamash (1994). A non-recursive method for solving the general discrete-time Riccati equations related to the $H_{\infty}$ control problem. International Journal of Robust and Nonlinear Control, Vol. 4, No. 4, pp. 503-519.

[4] Chen, B. M. (1996). Exact computation of infimum in discrete-time $H_{\infty}$-optimization using measurement feedback. Proceedings of the IFAC Automatic Control 13th Triennial World Congress, San Francisco, California, Vol. G: Education and Robust Control I, pp. 151-156.

[5] Chen, B. M., Y. Guo and Z. Lin (1996). Non-iterative computation of infimum in discretetime $H_{\infty}$-optimization and solvability conditions for the discrete-time disturbance decoupling problem. International Journal of Control, Vol. 65, No. 3, pp. 433-454.

[6] Commault, C., and J. M. Dion (1982). Structure at infinity of linear multivariable systems: A geometric approach. IEEE Transactions on Automatic Control, Vol. 27, pp. 693696.

[7] Franklin, G. F., J. D. Powell, and M. Workman (1980). Digital Control of Dynamic Systems. Second Ed., Addison-Wesley, Reading MA.

[8] Grizzle, J. W., and M. H. Shor (1988). Sampling, infinite zeros and decoupling of linear systems. Automatica, Vol. 24, No. 3, pp. 387-396.

[9] Kailath, T. (1980). Linear Systems. Prentice-Hall, Englewood Cliffs.

[10] Lin, Z. (1989). The Implementation of Special Coordinate Basis for Linear Multivariable Systems in MATLAB. Washington State University Technical Report Number ECE0100, Pullman, Washington.

[11] MacFarlane, A. G. J., and N. Karcanias (1976). Poles and zeros of linear multivariable systems: A survey of the algebraic, geometric and complex variable theory. International Journal of Control, Vol. 24, pp. 33-74.

[12] Morse, A. S. (1973). Structural invariants of linear multivariable systems. SIAM Journal on Control \& Optimization, Vol. 11, pp. 446-465.

[13] Moylan, P. (1977). Stable inversion of linear systems. IEEE Transactions on Automatic Control, Vol. 22, pp. 74-78. 
[14] Owens, D. H. (1978). Invariant zeros of multivariable systems: A geometric analysis. International Journal of Control, Vol. 28, pp. 187-198.

[15] Saberi, A., and P. Sannuti (1990). Squaring down of non-strictly proper systems. International Journal of Control, Vol. 51, No. 3, pp. 621-629.

[16] Saberi, A., B. M. Chen and P. Sannuti (1991). Theory of LTR for nonminimum phase systems, recoverable target loops, recovery in s subspace - Part 1: Analysis. International Journal of Control, Vol. 53, pp. 1067-1115.

[17] Saberi, A, P. Sannuti and B. M. Chen (1995). $H_{2}$ Optimal Control. Prentice-Hall, London.

[18] Sannuti, P., and A. Saberi (1987). A special coordinate basis of multivariable linear systems - Finite and infinite zero structure, squaring down and decoupling. International Journal of Control, Vol. 45, No. 5, pp. 1655-1704. 